\title{
Concurrent chemoradiotherapy with paclitaxel and cisplatin for patients of locally advanced cervical squamous cell carcinoma: A single-arm phase II clinical study.
}

\author{
Jia-Hao Zhu ${ }^{1,2 \#}$, Qun-Chao Hu${ }^{2 \#}$, Ke Gu${ }^{2 *}$, Sheng-Jun $\mathrm{Ji}^{2}$, Chen Shen ${ }^{3}$, Ru Yang ${ }^{3}$, Jie Chen ${ }^{2}$, Zheng- \\ Cao Liu ${ }^{2}$, Yan $\mathrm{He}^{2}$, Jin-Chang $\mathrm{Wu}^{2}$ \\ ${ }^{1}$ Department of Oncology, Nanjing Medical University, Nanjing, PR China
}

${ }^{2}$ Department of Radiation Oncology, Nanjing Medical University Affiliated Suzhou Hospital, Suzhou Municipal Hospital, PR China

${ }^{3}$ Department of Obstetrics and Gynecology, Nanjing Medical University Affiliated Suzhou Hospital, Suzhou Municipal Hospital, PR China

\#These authors have equally contributed to this study.

\begin{abstract}
Objectives: A phase II study was conducted to investigate the safety and efficacy of a regimen of cisplatin (DDP) plus paclitaxel (PTX) concurrent with radiotherapy (RT) in patients with locally advanced cervical squamous cell carcinoma (LACSC).

Patients and methods: 34 patients with newly diagnosed LACSC confirmed by histopathology, with a Karnofsky performance status $\geq 60$, no prior cervical cancer treatment, and no chemotherapy contraindications were enrolled. Concurrent chemoradiotherapy (CCRT) was delivered to all cases. The chemotherapy consisted of two 3-week cycles of DDP $\left(30 \mathrm{mg} / \mathrm{m}^{2} / \mathrm{d}\right)$ for $3 \mathrm{~d}$ plus PTX $\left(135 \mathrm{mg} / \mathrm{m}^{2}\right)$ for $1 \mathrm{~d}$. Concurrent radiation (pelvic irradiation of 45 Gy/25 Fx (PTV); lesion boost of 5-10 Gy (GTV); total dose 70-80 Gy) was administered by 3-dimensional conformal radiotherapy (3DCRT; N=14) or intensitymodulated radiation therapy (IMRT; $N=20$ ). Short-term efficacy and 1, 2 and 3 y, overall survival (OS) rates, and adverse reactions were analyzed.

Results: The cohort of 34 patients (median age, $60 \mathrm{y}$; range, 35-74) included 4 stage Ib2 cases, 16 stage II cases, 10 stage III cases, and 4 stage IV cases. With a median follow-up period of 38 months, the overall response rate (ORR) was $28 / 34(82.4 \%)$, including a $12(35.3 \%)$ with a complete response (CR) rate and $16(47.1 \%)$ with a partial response (PR). The 1,2 and 3 y OS rates were $100 \%, 94.1 \%$, and $88.2 \%$, respectively. Grade III occurred in 12 patients $(35.3 \%)$, but no patients suffered from grade IV myelosuppression. Gastrointestinal tract reactions, vaginitis, proctitis, and cystitis were common by mild in all cases.

Conclusion: CCRT with DDP plus PTX produced an encouraging and OS rates and ORR with relatively tolerable hematological toxicity.
\end{abstract}

Keywords: Concurrent chemoradiotherapy, Paclitaxel, Cisplatin, Locally advanced cervical squamous carcinoma.

Accepted on April 05, 2018

\section{Introduction}

Cervical cancer is the second most common female malignant tumor after breast cancer, and is the second most common cause of death from cancer in women [1]. More than 50 percent of patients have been diagnosed with locally advanced cervical carcinoma (LACC) in the first visit, posing a challenge to the surgery. Pelvic concurrent chemoradiotherapy (CCRT) is recommended for LACC according to NCCN Guideline [2,3]. Cisplatin (DDP), one of the most effective agents for various cancers, is widely used in the treatment of cervix carcinoma
[4]. Several studies demonstrated that combination of DDP and radiotherapy significantly improved the clinical outcome of advanced cervical cancer, compared to radiotherapy alone. Thus, DDP-based CCRT was rapidly adopted in clinical practice for the standard treatment of LACC [5]. Recently, some novel chemotherapeutic regimens based on DDP for CCRT appeared, such as DDP plus 5-FU, DDP plus gemcitabine and DDP plus paclitaxel (PTX), etc. CCRT with PTX plus DDP was reported to be the superior choice of concurrent chemotherapy regimen for LACC, concerning to its better 5-y disease-free survival rate and good tolerance [6]. 
Yet, more prospective data is still needed to validate those outcomes. Locally advanced cervical squamous cell cancer (LACSC) accounts for more than $90 \%$ of LACC. In the present study, we conducted a prospective single-arm phase II trial to evaluate the efficacy and tolerability of the definitive CCRT with PTX and DDP regimen in patients with LACSC.

\section{Patients and Methods}

\section{Eligibility}

Eligible LACC patients admitted to our hospital in-between April 2012 to May 2015 were registered for possible participation in this study. Based on the inclusion criteria, a total of 34 patients were enrolled. The inclusion criteria were: newly diagnosed with histologically confirmed LACSC; age $<75 \mathrm{y}$; and a Karnofsky performance status $\geq 70$. The exclusion criteria were: metastatic disease; previous treatment for cervical cancer; and hepatic, renal, or cardiac dysfunction. Normal bone marrow, liver, and renal function were confirmed before treatment.

\section{Pre-treatment evaluation}

Before being enrolled, all patients would underwent physical examination, gynaecological examination, pelvic enhanced magnetic resonance imaging (MRI), chest/abdominal computed tomography (CT) scanning, ultrasonographic examination, 12-lead electrocardiogram, full blood counts and biochemistry tests. $18 \mathrm{~F}$-fluorodeoxy glucose positron emission tomography was required in need of ruling out patients with suspicious nodes or distant metastasis. Disease was staged according to the International Federation of Gynecology and Obstetrics (FIGO) staging systems.

\section{Treatment}

CCRT regimen applied in the present study was PTX plus DDP (TP) chemotherapy combined with radiotherapy. The first cycle of concurrent chemotherapy was started on the initial day of radiotherapy. Patients were given PTX (135 mg/m², intravenous, day 1) plus DDP $\left(30 \mathrm{mg} / \mathrm{m}^{2}\right.$, intravenous, day 1-3), repeated every $21 \mathrm{~d}$. Patients were allowed to continue the second course during radiotherapy with acceptable adverse effects. Pre-treatment before PTX was applied as required: 27 tablets of dexamethasone administered orally $(0.75 \mathrm{mg} / \mathrm{tablets})$ at 12 and $6 \mathrm{~h}$ before PTX treatment successively, while $600 \mathrm{mg}$ climetidine and $25 \mathrm{mg}$ phenergan injection given half an hour before PTX.

If grade III or higher hematological toxicity occurred or a nadir granulocyte count $<500 / \mathrm{mm}^{3}$ or platelet count $<50,000 / \mathrm{mm}^{3}$ were found and persisted, then chemotherapy, DDP and PTX, was suspended until recovery and the regimen dose reduced sequentially by $25 \%$. In addition, chemotherapy would be stopped if any of the following situations occurred: patients requiring a delay in therapy of $>2$ weeks or $>2$ dose reductions.

\section{Radio-therapeutic technique}

In our study, both external beam radiotherapy and brachytherapy were used. Patients underwent 3-dimensional conformal radiotherapy (3DCRT) or intensity-modulated radiation therapy (IMRT) with $6 \mathrm{MV}$ X-ray. Computerized imaging was used to define the target volumes. Details of radiotherapy plan were described as follows: $1.8 \mathrm{~Gy} /$ fraction, 5 fractions a week, 45 Gy in 25 fractions to the planning target volume (PTV) in 5 weeks. And then boost was given with 5-10 Gy to gross tumor lesion. Patients would receive after-loading radiotherapy with $192 \mathrm{Ir}$ at the late period of external beam radiotherapy according to the tumor regression. The prescribed dose of brachytherapy was 20-30 Gy in 4-6 fractionstwice a weekadded up to a total dose of 70-80 Gy for the para-cervical reference point, point A (Table 1).

Table 1. Treatment plan.

\begin{tabular}{|c|c|c|c|c|c|c|c|c|c|}
\hline \multirow[t]{3}{*}{ Days } & \multicolumn{6}{|c|}{ Radiotherapy } & \multicolumn{3}{|c|}{ Brachytherapy } \\
\hline & 1 & 7 & 14 & 21 & 28 & 35 & 42 & 49 & 56 \\
\hline & $\mathrm{P}$ & & & PT & & & & & \\
\hline
\end{tabular}

PT=Cisplatin $30 \mathrm{mg} / \mathrm{m}^{2} / \mathrm{d}, \mathrm{d}$ 1-3+Paclitaxel $135 \mathrm{mg} / \mathrm{m}^{2} / \mathrm{d} 1$.

Plan optimization was based on the dose-volume histogram (DVH) as follows: (1) The prescribed isodose curve covered 95\% of the PTV; (2) The $95 \%$ isodose curve covered $99 \%$ of the PTV; (3) The maximum dose within the PTV was limited to $110 \%$ of the prescribed dose; (4) Inhomogeneity corrections were carried out. Dose constraints to the organs at risk (OAR) were as follows: (1) The maximum dose on spinal cord $\leq 40$ Gy; (2) Bladder V45<80\%; (3) Rectum V45<80\%; (4) Caput femoris V30<20-50\%; (5) Colon V40<80\%; (6) Anal tube $\mathrm{V} 30<80 \%$.

\section{Follow-up evaluations}

During treatment, blood cell counts were assessed biweekly. Patients were screened for other adverse symptoms daily. Toxicity was evaluated according to the common toxicity criteria version 3.0 .

Short-term efficacy was evaluated according to Response Evaluation Criteria in Solid Tumors (RECIST) criteria. Briefly, disappearance of all target lesions with no new lesions appearing for $>4$ weeks was considered a complete response (CR). $A \geq 50 \%$ decrease in target lesion volume with no new lesions appearing $>4$ weeks was considered a partial response (PR). A decrease in target lesion volume (s) of $<50 \%$ or an increase target lesion volume of $<25 \%$ was considered stable disease (SD). Finally, an increase in target lesion volume(s) of $>25 \%$ and the appearance of new lesions was considered progressive disease (PD). The sum of CR and PR cases divided by the total number of cases constituted the objective response rate $(\mathrm{ORR})$.

After completion of therapy, the patients were observed every 3 months for $2 \mathrm{y}$, and then on a half-yearly basis. Follow-up 
Concurrent chemoradiotherapy with paclitaxel and cisplatin for patients of locally advanced cervical squamous cell carcinoma: A single-arm phase II clinical study

examinations included functional assessments of bone marrow, the liver, and the kidneys. Electrocardiogram, chest CT, and ultrasonography or CT/MRI of the abdomen and pelvis were carried out. Biopsies of suspicious areas detected by imaging were performed. The last patient follow-up was in October of 2017. The median follow-up time was 38 months (range, 24-42).

\section{Ethical considerations}

This prospective phase II study was carried out with strict adherence to the Declaration of Helsinki. The study protocol was reviewed and approved by the Ethics Committee of Suzhou Municipal Hospital (East Branch). Written informed consent was obtained from all participants.

\section{Statistical analysis}

We analyzed short-term efficacy, and 1, 2, and 3-y overall survival (OS) rates, and toxicity frequencies. OS rates were subjected to Kaplan-Meier modeling in SPSS (version 11.0). All endpoints were observed from the first day of treatment until death or the last follow-up evaluation (Figure 1).

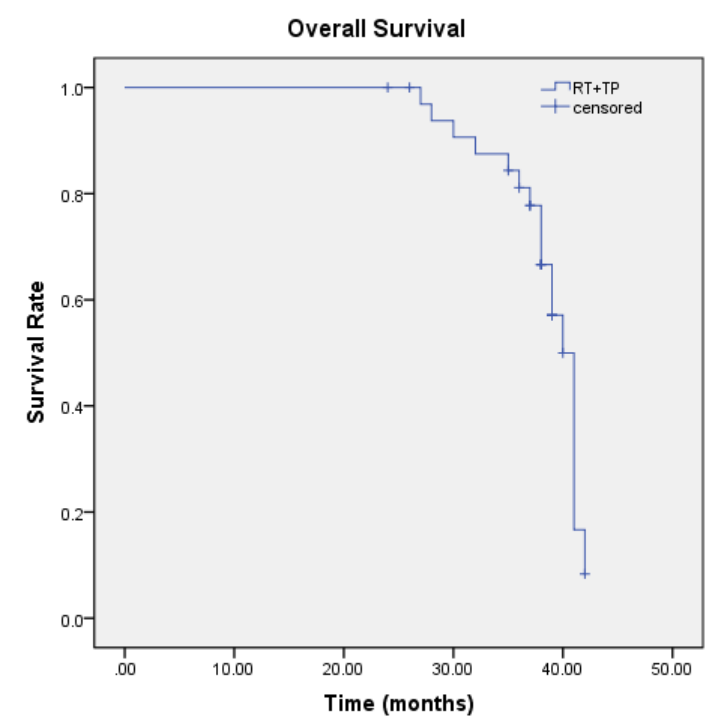

Figure 1. OS curve for the study cohort $(N=34)$.

\section{Results}

\section{Demographic variables of the patients}

From April 2012 to May 2016, we enrolled 34 patients to this study. Mean age of these patients was $60 \mathrm{y}$ (range 35 to $74 \mathrm{y}$ ). The demographics and clinicopathologic characteristics were summarized in Table 2. All patients completed radiotherapy and 2 cycles of concurrent TP chemotherapy per protocol. The last follow-up time was October 2017. The median follow-up time was 38 months (range, 24 to 42 months).

Table 2. Patient Characteristics $(N=34)$.

\begin{tabular}{|c|c|}
\hline \multicolumn{2}{|l|}{ Age $(y)$} \\
\hline$\leq 60$ & 18 (52.9) \\
\hline$\geq 60$ & $16(47.1)$ \\
\hline Median & $60(35-74)$ \\
\hline \multicolumn{2}{|l|}{ FIGO } \\
\hline IB2 & $4(11.8)$ \\
\hline IIA2 & $6(17.6)$ \\
\hline IIB & $10(29.4)$ \\
\hline IIIA & $4(11.8)$ \\
\hline IIIB & $6(17.6)$ \\
\hline IVA & $4(11.8)$ \\
\hline \multicolumn{2}{|c|}{ Pathological grade } \\
\hline Low & $22(64.7)$ \\
\hline Middle & $10(29.4)$ \\
\hline High & $2(5.9)$ \\
\hline \multicolumn{2}{|c|}{ Radiotherapy } \\
\hline 3DCRT & $14(41.2)$ \\
\hline IMRT & $20(58.8)$ \\
\hline \multicolumn{2}{|c|}{$\begin{array}{l}\text { FIGO: International Federation of Gynecology and Obstetrics; 3DCRT: 3- } \\
\text { Dimensional Conformal Radiotherapy; IMRT: Intensity-Modulated Radiation } \\
\text { Therapy. }\end{array}$} \\
\hline
\end{tabular}

\section{OS and ORR}

Until the last follow-up, 28 patients showed no evidence of disease progression after the regimen of TP CCRT treatment. The ORR was $82.4 \%$ (Table 3 ). The $1,2,3$ y OS rates were $100 \%, 94.1 \%$, and $88.2 \%$, respectively (Table 4 ). The median survival time of our study was 38.6 months (95\% CI: 37.154-40.028).

Table 3. Locally advanced cervical carcinoma short-term efficacy of patients $(N=34)$.

\begin{tabular}{ll}
\hline Treatment & No. of patients $(\mathbf{n}(\%))$ \\
\hline CR & $12(35.3)$ \\
\hline PR & $16(47.1)$ \\
\hline SD & $4(11.8)$ \\
\hline PD & $2(5.9)$ \\
\hline Response rate (CR+PR) & $28(82.4)$ \\
\hline
\end{tabular}

CR: Complete Response; PR: Partial Response; SD: Stable Disease; PD: Progressive Disease.

Table 4. 1 y, 2 y and 3 y overall survival rates of patients.

\begin{tabular}{ll}
\hline Year & Survival rate $(\%)$ \\
\hline 1 & 100 \\
\hline
\end{tabular}




\begin{tabular}{ll}
\hline 2 & 94.1 \\
\hline 3 & 88.2 \\
\hline
\end{tabular}

\section{Adverse events}

Toxicities of the treatment in our trial were summarized in Table 5. Previous studies showed that the most frequent acute adverse events were grade III or IV neutropenia and leukopenia. But in the present trial, grade III myelosuppression was $35.3 \%$ (12/34) and no patient suffered from grade IV bone marrow suppression. Non-hematological adverse events were mild and well tolerated. Nausea/vomiting were common during time of chemotherapy, with grade I (26/34) and grade II (8/34) gastrointestinal reactions. Radiotherapy-related adverse events included mainly vaginitis (28/34 (grade I), 6/34 (grade II)), proctitis (28/34 (grade I), 6/34 (grade II)) and cystitis (30/34 (grade I)). No grade III or IV radiotherapy-induced toxicity was observed. And there was no treatment relevant toxicity for liver, kidney or heart occurred.

Table 5. Treatment toxicities of patients $(N=34)$.

\begin{tabular}{lll}
\hline Toxicities & Grade & No. of patients (n (\%)) \\
\hline Radiation-induced vaginitis & I & $28(82.4)$ \\
\cline { 2 - 3 } & II & $6(17.7)$ \\
\hline Radiation-induced proctitis & I & $28(82.4)$ \\
\cline { 2 - 3 } & II & $6(17.7)$ \\
\hline Radiation-induced cystitis & I & $30(88.2)$ \\
\hline Gastrointestinal tract reaction & I & $26(76.5)$ \\
\cline { 2 - 3 } & II & $8(23.5)$ \\
\hline Bone marrow suppression & I-II & $22(64.7)$ \\
\cline { 2 - 3 } & III & $12(35.3)$ \\
\hline
\end{tabular}

\section{Discussion}

The present study showed that CCRT with DDP plus PTX had good short-term efficacy and encouraging OS rates with relatively tolerable hematological toxicity. The presently examined multi-drug regimen of TP in CCRT for LACC has previously been shown to yield superior outcomes relative to other DDP-based regimens [6]. The use of CCRT has increased the 5-y OS rate of patients with LACC significantly, by up to $6 \%[7]$.

Traditionally, LACC has included FIGO stage IIb-IVa phase lesions, though many oncologists now consider FIGO stages $\mathrm{Ib} 2$ and IIa2 to be LACC, expanding the proportion of cervical cancer cases classified as LACC. Consequently, LACC treatment efficacy is a major determinant of cervical cancer prognosis as a whole.

PTX, a taxane compound, has been shown to have high efficacy against solid tumors, especially epithelial ovarian cancer, lung, and breast cancer tumors [8]. PTX cytotoxicity of human cervical cancer cells has been shown to involve inhibition the Raf-1 kinase activity. PTX inhibits mitosis by binding tubulin, a structural protein that is necessary for cell division, keeping tumor cells stuck in the G2 and M phases. PTX also appears to contribute to re-oxidation of hypoxic tumor cells [9].

Thus, it may function as radiation sensitizer, making it appropriate for use with RT [10]. In a phase II randomized trial comparing RT with concurrent weekly DDP or PTX in patients with LACC, Geara et al. did not find a significant advantage of PTX over DDP [11].

Moore et al. demonstrated that CCRT with TP was superior to CCRT with DDP alone in terms of ORR and progressive free survival in patients with LACC [12]. Additionally, more recently, Umayahara et al. documented favorable antitumor activity of CCRT with TP [13].

In this study, we found that CCRT with $\mathrm{TP}$ regimen provided LACSC patients with 1,2 , and 3 -y survival rates of $100 \%$, $94.1 \%$ and $88.2 \%$, respectively. The median survival time was 38.6 months (95\% CI: 37.2-40.0). The ORR of CCRT in our study was $82.4 \%$, which was comparable to previous reports of $80 \%-100 \%$.

Due to additional cycle of neoadjuvant chemotherapy before CCRT, Miglieta et al. observed full complete clinical response rate and maintained local control [8]. It was assumed that high dose of PTX (175 mg/m $\mathrm{m}^{2}$ every 3 weeks for four cycles) in chemotherapy regimen would contribute to favourable tumor regression in that study. Varghese et al. [14,15] presented relatively lower response rate of $88 \%$, even with dose-dense concurrent chemotherapy regimen of cisplatin $30 \mathrm{mg} / \mathrm{m}^{2}$ and paclitaxel $40 \mathrm{mg} / \mathrm{m}^{2}$ for 4 weeks along with radiotherapy.

Though the authors considered no extra benefit with additional use of paclitaxel in the chemoradiation, we should notice that high rate of lost on follow-up might increase reporting biases in that study. Similarly, $\mathrm{Ke}$ et al. launched concurrent chemotherapy with weekly application of DDP $\left(20 \mathrm{mg} / \mathrm{m}^{2}\right)$ and taxotere $\left(35 \mathrm{mg} / \mathrm{m}^{2}\right)$ for $4-5$ weeks combining with daily radiation therapy (total dose of 65-80 Gy).

In our study, chemotherapy consisted of DDP $\left(30 \mathrm{mg} / \mathrm{m}^{2}\right.$, day 1-3) and PTX (135 mg/m², day 1$)$, every 3 weeks for 2 cycles, and radiotherapy with total dose of 70-80 Gy to point A. High proportion of bulky tumor (6/34) and extensive invasion of local regional area (FIGO stage IIIA-IVA 14/34) might lead to mild early remission. In the study of Negi et al. [16], DDP (40 $\left.\mathrm{mg} / \mathrm{m}^{2}\right)$ and 5 -Fluorouracil $\left(500 \mathrm{mg} / \mathrm{m}^{2}\right)$ were provided every $21 \mathrm{~d}$ to LACC patients, with a total radiation dose of 81 Gy to point A. And the response rate of the cohort was only $80 \%$.

All 34 patients finished two full TP courses with a full RT course. The median RT time in our study was $62 \mathrm{~d}$, similar to the 63-d median reported by Miglietta et al. [8]. Adverse effects in our trial were generally tolerable and mild. Most concerning was Grade III bone marrow suppression in slightly more than a third of the cohort. 
No grade IV hematotoxicity was observed. Although gastrointestinal tract reactions, vaginitis, proctitis, and cystitis were common, they were mild (grade I or II) in all cases. Ke et al.'s regime produced a $82.1 \%$ rate of myelosuppression (grade I, II, or III; no grade IV) [15]. A third of the patients in Miglietta et al.'s [8] study showed grade 3 or 4 hematological toxicity, whereas slightly more than three quarters of the patients in Sood et al.'s [17] study experienced grade III or IV acute hematological toxicity.

Comparing the toxicities of CCRT with DDP plus 5fluorouracil versus CCRT with DDP plus PTX in patients with LACC, Sol et al. [18] found that the latter group had a higher frequency of severe (grade III or IV) leukopenia $(79.2 \% \mathrm{vs}$. $11.1 \%)$ but a lower frequency of severe nausea $(14.6 \% \mathrm{vs}$. $33.3 \%)$.

In conclusion, a CCRT regime of PTX plus DDP produced satisfactory short-term efficacy with relatively mild side effects. This study was limited by a small sample size and short follow up. Further observation of the safety and efficacy of this regime is needed to ascertain its long-term effects.

\section{Acknowledgement and Funding}

This study was supported by the Research and Development Key Program of Jiangsu Province (Grant BE2015645), the Scientific Research Project of Health Department of Jiangsu Province (Grant Z201413), and Suzhou Key Medical Center Program (Grant szzx201506).

\section{Competing Interests}

The authors have no financial competing interests to declare do not have any political, personal, religious, ideological, academic, intellectual, or commercial competing interests to declare related to the manuscript.

\section{References}

1. Shi JF, Qiao YL, Smith JS. Epidemiology and prevention of human papillomavirus and cervical cancer in China and Mongolia. Vaccine 2010; 28: 2573-2574.

2. Rose PG, Bundy BN, Watkins EB. Concurrent cisplatinbased radiotherapy and chemotherapy for locally advanced cervical cancer. N Engl J Med 1999; 340: 1144-1153.

3. Vale C, Tierney J, Stewart L. Reducing uncertainties about the effects of chemoradiotherapy for cervical cancer: a systematic review and meta-analysis of individual patient data from 18 randomized trials. J Clin Oncol 2008; 26: 5802.

4. Li R, Liu GZ, Luo SY. Cyclin I promote cisplatin resistance via Cdk5 activation in cervical cancer. Eur Rev Med Pharmacol Sci 2015; 19: 4533-4541.

5. Au-Yeung G, Mileshkin L, Bernshaw DM. Radiation with cisplatin or carboplatin for locally advanced cervix cancer: the experience of a tertiary cancer centre. J Med Imaging Radiat Oncol 2013; 57: 97-104.
6. Fu ZZ, Li K, Peng Y. Efficacy and toxicity of different concurrent chemoradiotherapy regimens in the treatment of advanced cervical cancer: A network meta-analysis. Medicine 2017; 96: e5853.

7. Eifel PJ. Concurrent chemotherapy and radiation therapy as the standard of care for cervical cancer. Nat Clin Pract Oncol 2006; 3: 248-255.

8. Miglietta L, Franzone P, Centurioni MG, Boni L, Tacchini L, Cosso M. A phase II trial with cisplatinpaclitaxel cytotoxic treatment and concurrent external and endocavitary radiation therapy in locally advanced or recurrent cervical cancer. Oncology 2006; 70: 1924.

9. Milas L, Hunter NR, Mason KA. Role of reoxygenation in induction of enhancement of tumor radioresponse by paclitaxel. Cancer Res 1995; 55: 3564-3568.

10. Therasse P, Arbuck SG, Eisenhauer EA, Wanders J, Kaplan RS, Rubinstein L. New guidelines to evaluate the response to treatment in solid tumors. J Natl Cancer Inst 2000; 92: 205216.

11. Geara FB, Shamseddine A, Khalil A. A phase II randomized trial comparing radiotherapy with concurrent weekly cisplatin or weekly paclitaxel in patients with advanced cervical cancer. Radiation Oncol 2010; 5: 84.

12. Moore DH, Blessing JA, McQuellon RP. Phase III study of cisplatin with or without paclitaxel in stage IVB, recurrent, or persistent squamous cell carcinoma of the cervix: a gynecologic oncology group study. J Clin Oncol 2004; 22: 3113-3119.

13. Umayahara K, Takekuma M, Hirashima Y. Phase II study of concurrent chemoradiotherapy with weekly cisplatin and paclitaxel in patients with locally advanced uterine cervical cancer: The JACCRO GY-01 trial. Gynecol Oncol 2016; 140: 253-258.

14. Varghese SS, Ram TS, Pavamani SP. Concurrent chemoirradiation with weekly cisplatin and paclitaxel in the treatment of locally advanced squamous cell carcinoma of cervix: a phase II study. J Cancer Res Therap 2014; 10: 330-336.

15. Ke QH, Zhou SQ, Du W. Early efficacy of taxotere and cisplatin chemo-radiotherapy for advanced cervical cancer. APJCP 2012; 13: 617-619.

16. Negi RR, Gupta M, Kumar M. Concurrent chemoradiation in locally advanced carcinoma cervix patients. J Cancer Res Therap 2010; 6: 159.

17. Sood BM, Gorla GR, Garg M, Anderson PS, Fields AL, Runowicz CD, Goldberg GL, Vikram B. Extended-field radiotherapy and high-dose-rate brachytherapy in carcinoma of the uterine cervix: clinical experience with and without concomitant chemotherapy. Cancer 2003; 97: 1781-1788.

18. Sol ES, Lee TS, Koh SB. Comparison of concurrent chemoradiotherapy with cisplatin plus 5-fluorouracil versus cisplatin plus aclitaxel in patients with locally advanced cervical carcinoma. J Gynecol Oncol 2009; 20: 28-34. 


\section{*Correspondence to}

$\mathrm{Ke} \mathrm{Gu}$

Department of Radiation Oncology

Nanjing Medical University Affiliated Suzhou Hospital

Suzhou Municipal Hospital

PR China 